\title{
ESTUDIO PRELIMINAR DEL USO DE LAS REDES SOCIALES EN LOS JÓVENES ECUATORIANOS
}

\section{PRELIMINARY STUDY OF THE USE OF SOCIAL NETWORKS IN THE ECUADORIAN YOUNG PEOPLE}

\author{
JAVIER GUAÑA-MOYA ${ }^{1}$ \\ EDWIN QUINATOA-AREQUIPA ${ }^{2}$ \\ LENIN LARREA-TORRES ${ }^{3}$
}

Recibido: 27 de septiembre de 2017

Aceptado: 31 de enero de 2018

\footnotetext{
${ }^{1}$ Pontificia Universidad Católica del Ecuador, (eguana953@puce.edu.ec).

2Universidad Técnica del Cotopaxi, (edwin.quinatoa@utc.edu.ec).

${ }^{3}$ Instituto Ecuatoriano de Seguridad Social, (llarreat@iess.gob.ec).
} 
ill|lin

\footnotetext{
224
} 


\title{
ESTUDIO PRELIMINAR DEL USO DE LAS REDES SOCIALES EN LOS JÓVENES ECUATORIANOS
}

\author{
PRELIMINARY STUDY OF THE USE OF SOCIAL \\ NETWORKS IN THE ECUADORIAN YOUNG PEOPLE
}

Javier Guaña-Moya, Edwin Quinatoa-Arequipa, Lenin Larrea-Torres

Palabras Clave: Internet, comunicación, redes sociales, privacidad, web.

Keywords: Internet, communication, social networks, privacy, web.

\section{RESUMEN}

El presente artículo describe la importancia que ha adquirido hoy en día el uso de la Internet y las redes sociales, así como también el análisis de los efectos de las relaciones interpersonales a través de una comunicación interactiva y online, logrando así que personas tímidas se expresen de forma libre y tranquila, con una mejor confianza en el ser humano y la aceptación en la sociedad actual. Por ello se puede especificar que la sociedad ha evolucionado en torno a la tecnología y calidad de vida, de esta forma el desarrollo tecnológico ha provocado grandes cambios económicos, culturales, sociales, educativos, etc., por ello se describe que el modo de comunicarse a través de redes sociales ha sustituido la sociabilización humana, es decir, desarrolla comunicaciones cara a cara, excluyéndoles de emociones tangibles; también ha permitido la comunicación en lugares distantes, de tal forma que nuestras vidas se ven relacionadas por 
personas que conocemos a través de una red social o chat, logrando así acortar tiempos y distancias, alcanzar una comunicación interactiva y de forma real, basada en los medios y aplicaciones tecnológicas existentes en el mercado.

\section{ABSTRACT}

This article describes the importance that the use of the Internet and social networks has acquired today, as well as the analysis of the effects of interpersonal relationships through interactive and online communication, thus making shy people express themselves Free and quiet way, achieving a better confidence in the human being and acceptance in today's society. Therefore, it can be specified that society has evolved around technology and quality of life, in this way technological development has caused great economic, cultural, social, educational, etc. changes, for this reason it is described that the way of communicating to through social networks it has replaced human socialization, that is, it develops face-to-face communications, excluding them from tangible emotions; It has also allowed communication in distant places, in such a way that our lives are related by people we know through a social network or chat, thus shortening times and distances, achieving an interactive communication in a real way, based on the media and technological applications existing in the market.

\section{INTRODUCCIÓN}

Las nuevas tecnologías de la información y comunicación, también denominadas TICs, han formado una nueva forma de comunicación que permite que los flujos de información sean rápidos, amplios, reversibles y accesibles, logrando así que la transferencia de información sea de forma inmediata y todos los saltos tecnológicos producidos sean de forma acelerada, mejorando la comunicación y la interacción a través del Internet. Moya, E. J. G., Escobar, A. G. A., \& Remache, K. J. O. (2015).

Esta etapa ha marcado los procesos productivos y de comunicación a nivel mundial, denominándose Revolución Digital, por lo cual se ha designado inexorable confluencia de los sectores estratégicos implicados en el nuevo escenario comunicativo, y solo aquellos 
actores que sean capaces de interpretar con facilidad los indicadores que emergen, podrán ser protagonistas del cambio (Campos, 2013).

Piscitelli A, Adaime I, Binder I. (2010), describen que gran parte de usuarios han integrado la era tecnológica en su vida diaria, convirtiéndose en un elemento de interacción social especialmente para los jóvenes donde pueden compartir información, fotos, videos o mensajes instantáneos.

La interactividad se define como la capacidad que tiene el usuario de "preguntar" al sistema, y sentar las bases para recuperar la información de la forma deseada. El emisor, no envía un mensaje unidireccional, sin capacidad de respuesta, y el receptor puede tomar decisiones, configurar su propio mensaje, así como dialogar, de una forma u otra con el emisor (interactividad sujeto / objeto), Armañanzas, E., Díaz, J., \& Meso, K. (1996).

La interactividad, es la retroalimentación que se efectúa en el proceso de comunicación entre el emisor y el receptor, donde se intercambia información y se recibe respuesta, así como también los medios digitales ofrecen herramientas interactivas desarrolladas para comunicarse con los receptores que fa- vorecen la comunicación y resultan más rápidas y sencillas que las tradicionales (cartas, llamadas).

La forma tradicional de comunicarse de los jóvenes cara a cara es poco utilizada hoy en día, de la misma forma la comunicación basada en texto, mensajería, y se prefiere el método de comunicación instantánea. La Internet y las redes sociales son cada vez más utilizadas, especialmente en los jóvenes por que han crecido juntos con la tecnología, por ello se les ha denominado nativos digitales y su uso es cada vez más frecuente. Cabe describir que las redes sociales son simplemente un canal que les permite relacionarse con personas que quizá en la vida real no hubiera sido posible. En el caso de jóvenes tímidos o extrovertidos quienes tienen una personalidad muy vulnerable frente a otras personas, tienen la oportunidad de entablar relaciones sociales, de ser aceptados o valorados de forma igualitaria, permitiéndoles romper la barrera de la timidez y el paradigma de la comunicación social, por lo cual el objetivo de este artículo es establecer los efectos que tienen las redes sociales en los jóvenes ecuatorianos por medio del análisis de contenido de información. 


\section{METODOLOGÍA}

La investigación se realizó a través de los procesos cuantitativos (análisis de contenido), efectuando un estudio selectivo de información que revele el uso de la Internet y las redes sociales en los ecuatorianos, también se generó un análisis de perfiles de la red social por medio de datos estadísticos y por medios de comunicación que cumplían con las carac- terísticas de esta investigación. En cuanto a la información obtenida se inclinó al uso de las redes sociales en la población ecuatoriana y algunos datos estadísticos referente al uso de la tecnología y del uso de la Internet, se tomó como base la descripción estadística de Internet World Stats, misma que tiene bases de datos nacionales e internacionales.

\section{RESULTADOS}

\section{Uso de la Internet}

En Sudamérica se estima que en el 2014, el uso de la Internet fue $54.7 \%$ y que para el 2016, se incrementó en un $57 \%$, por ello la nueva cultura digital implica que Internet sea parte de la vida cotidiana, mientras que las TICs se conviertan en herramientas para desarrollar actividades como la compra y venta digital, incluyendo a todos los individuos en la sociedad; estos nuevos procesos permiten generar nuevas tendencias del uso de las tecnologías, por ello las redes sociales permiten una nueva forma de comunicarse, de relacionarse y de crear comunidades virtuales, basados en la interacción directa de la Internet y de las diferentes aplicaciones en línea, (Guaña-Moya, E. J., Quinatoa-Arequipa, E., \& Pérez-Fabara, M. A. (2017).
Según, Stats, I. W. (2017), en Latinoamérica los usuarios conectados a la Internet hasta el 30 de junio del 2017 son 404.269.163 y el crecimiento del 2000 2017 es de $2.137,4 \%$ y los suscriptores en Facebook al 30 de junio 2017 son 257.242.500.

Ecuador tiene una población aproximada de 16,625,776 a inicios del 2017, los usuarios conectados a Internet a marzo 2017 son 13.471 .736 , esto quiere decir que tiene el $81.0 \%$ de penetración, según Supertel, y los suscriptores en $\mathrm{Fa}$ cebook al 30 de junio 2017 son 9.700.000, con una tasa de penetración de $58.3 \%$.

Según Acosta, Eva. (2017), el Instituto Nacional de Estadística y Censos INEC, (2017) describe que Ecuador tuvo 4,2 millones de personas que usaron en el 2016 las redes sociales en sus teléfonos 
inteligentes, también describe que este acontecimiento se basa en que el número de usuarios de Smartphone creció un $700 \%$ en los últimos cinco años, por ello se puede describir que, de acuerdo con el INEC, del 2011 al 2016 se incrementó de 522.000 personas a 4,5 millones de usuarios de teléfonos inteligentes.

En Ecuador, aproximadamente un $98 \%$ de personas que están sobre los 12 años de edad está registrado a una red social (Facebook). De acuerdo con los resultados de la Encuesta de Condiciones de vida elaborada por el Instituto Nacional de Estadísticas y Censos (INEC). Esto refleja la gran acogida de la nueva tecnología y sus formas de comunicación en los jóvenes. Para conseguir la información se efectuó una encuesta a 29052 viviendas de las 24 provincias del país, según el INEC. La encuesta se realizó a los miembros del hogar de 12 años de edad en adelante.

A nivel nacional, los resultados son que un $41,4 \%$ de las personas que realizaron la encuesta posee una cuenta en una red social; entre las más utilizadas están: Facebook, Twitter, YouTube, WhatsApp, YouTube, Skype y otras redes de acuerdo INEC.

A pesar de las políticas de privacidad y empleo de la imagen, acceso de menores de edad, los contenidos y más, las redes sociales se vuelven más populares en el mundo y necesarias.
Según Foon-Hew, K., (2011), sugieren que Facebook hasta ahora tiene muy poco uso educativo, que los estudiantes usan Facebook principalmente para mantenerse en contacto con personas conocidas, y que los estudiantes tienden a divulgar más información personal sobre ellos mismos en Facebook y que la evolución de las redes sociales como Facebook, MySpace, Linkedln, Twitter, entre otras, han cautivado a millones de usuarios ya que las redes son estructuras compuestas de un grupo de personas que se relacionan por amistad, parentesco o algún interés en particular, creadas libre y gratuitamente que funcionan a base de un perfil de usuario.

\section{Utilización de las redes sociales}

De acuerdo con los datos estadísticos publicados por Cobertura Digital, podemos comprobar que en Ecuador ha bajado el porcentaje de uso de redes sociales de jóvenes de 13 a 18 años que antes representaba el $28 \%$ en el 2014 y ahora el $18 \%$ para fines de 2016 e inicios 2017 y su migración a Instagram, Snapchat.

Mientras que los usuarios comprendidos entre los 18 y los 34 años es la que más ha crecido pasando a representar a más del $60 \%$ de la población del Ecuador en esta red social. 


\section{Efectos y causas de las redes sociales}

Gracias a las características propias de cada red social, las mismas que la hacen atractivas a las masas de jóvenes, se tiene un falso concepto en las personas adultas que resultan contraproducentes para los menores de 21, sin embargo, los jóvenes son responsables en el uso de las redes sociales, aunque hay excepciones y conviene educar el uso y empleo de esta herramienta tecnológica.

El análisis de los procesos económicos es un aspecto fundamental para la comprensión de su funcionamiento, ya que la teoría de las redes sociales muestra una gran potencialidad en este sentido, por lo que permite una notable simplificación del esquema de relaciones intersectoriales y su utilización posibilita un conocimiento en detalle de las relaciones de un sector con su entorno, (García Muñiz, A. S., \& Ramos Carvajal, C. (2003).

El uso excesivo de la Internet y las redes sociales se centra en la utilización y el empleo de las mismas por las personas más vulnerables (jóvenes), por lo cual puede asumirse como un problema cuando se evada la realidad, el tiempo de uso y se vuelva una adicción incontrolable por cada usuario de dichas actividades.

En la adolescencia y juventud esto se presenta como un grave pro- blema, ya que las redes sociales pueden ofrecerse como un espacio libre, en el cual los jóvenes con una personalidad vulnerable, dificultades familiares, temperamento débil, circunstancias de estrés, fracaso escolar, frustraciones afectivas, competitividad en procesos académicos / deportivos y con pocas relaciones sociales puede volverse un seguidor acérrimo a las redes sociales y difundir las acciones que no podrían hacerlo de forma normal.

Morduchowicz, R., Marcon, A., Sylvestre, V., \& Ballestrini, F. (2012), describen que alrededor de los 6 últimos años, el uso de la tecnología de los adolescentes en casa bordea un incremento del $45 \%$ de uso, esto ha permitido un análisis de uso de los equipos, por lo cual 4 de cada 10 adolescentes estén conectados las 24 horas al día; 5 de cada 10 están conectados hasta que van a dormir y solo 1 de cada 10 se conecta menos de 3 horas.

Por todo lo expuesto, se puede describir que la mayoría de jóvenes toman las Redes Sociales para la creación de una nueva identidad, preguntándose primero quién soy en el mundo digital y cómo me ven el resto de la comunidad. Esta nueva identidad permite que cualquier persona conozca sus actividades diarias, educativas, familiares, entre otras; logrando así autonomía en sus actos y compartiendo información indiscriminada, sin restricción y sin limitaciones. 
Esta nueva identidad obtenida en las Redes Sociales, permite fortalecer su confianza y autoestima, logrando así una comunicación libre, probando nuevas conductas y sin límites de acción y reacción en la sociedad digital, buscando así la visibilidad con sus amigos virtuales y diversos internautas.

Esta visibilidad y nueva identidad, lograrán la popularidad deseada en el mundo de la web, así como también pertenecer a una red social sin restricción y para marcar su propia identidad, sin importar el sentido de pertenencia en la información compartida y sin limitaciones en la información que desea mostrar. Todo esto con el deseo de ser popular con todos y ganarse un espacio en la sociedad digital.

Es importante describir que las redes sociales permiten que cada persona tenga una identidad propia y una sociedad digital amplia e inmersa en todo lo que se desee decir, compartir o comunicar; por lo que cada uno de los implicados se sienten libres, autónomos e independientes en realizar cualquier actividad y con la libertad de ser visibles, de verse y ser vistos por cualquier persona, de sentirse aceptados por una sociedad inherente y ser parte de esa comunidad tan deseada, para lograr popularidad y probarse que pueden construir un nuevo mundo virtual, basado en su identidad y su liber- tad en la toma de decisiones de cada individuo.

\section{Educación para un buen uso de las Redes Sociales}

El avance tecnológico, la Internet y las redes sociales, han permitido que el ser humano desarrolle nuevas habilidades y destrezas en los procesos de aprendizaje, así como también han llevado a una comunidad aislada, perdiendo en muchos casos la privacidad de la información compartida y de un rol que muchos toman para lograr su identidad deseada, logrando así conquistar sus miedos y generando un mejoramiento en su autoestima, sin darse cuenta que la persona que comparte, escribe, aconseja o negocia no es quien podría ser en la vida real, por ello es importante tener en cuenta algunas sugerencias que se deberían dar a los jóvenes que comienzan sus vidas en las redes sociales y la comunidad de la Internet.

- No incluir información personal relevante en los perfiles de las redes sociales.

- Abstenerse de subir fotos o imágenes privadas ya que pueden vulnerar su privacidad

No subir información, fotos y videos que no le pertenezcan. Es importante respetar la privacidad y la autoría de cada individuo. 
- Tener cuidado con la información que se publica porque datos relevantes como horarios de actividades que realiza cotidianamente pueden ser tomados para secuestros, robos, entre otros.

- Explicar que toda la información que se sube a la web es vulnerable y de dominio público, por lo que una vez subido a la Internet se podrá compartir o visualizar por todos los internautas, por lo que la información es muy difícil de borrar.

- Concientizar que todos tenemos derecho a la intimidad.

- No compartir información que no sea propia, ya que estamos interrumpiendo en los derechos de otras personas y esto podría llevarnos a tener problemas civiles e incluso penales.
- Utilizar la Internet con ética y cuidarse del acoso, bullying o cualquier tipo de violencia que se pueda generar en dichos medios.

Cuando se desee investigar o consultar en la web, se deberán utilizar fuentes confiables, chequear su contenido y siempre se deberá citar al autor del libro, artículo, sitio web, etc.

- Explicar a los jóvenes qué son sitios no deseados, pornografía, pedofilia, racismo, etc.

- Llevar un control del uso de la Internet y de los equipos tecnológicos, logrando así un momento para navegar en familia y conocer nuevas experiencias, obtener el diálogo y la confianza en todo momento y construyendo un código familiar, basado en respeto y valores que todos deben tener.

\section{CONCLUSIONES}

Tras realizar la investigación, basados en datos estadísticos concluimos que las redes sociales son páginas estructuradas, utilizadas especialmente por usuarios jóvenes entre 16 y 24 años, y una de las páginas lideres es Facebook y el perfil del internauta está definido por persona que utiliza la red principalmente para comunicarse, informarse, educarse y trabajar.
El porcentaje de usuarios ecuatorianos que poseen una red social es del $58.6 \%$, mientras que el porcentaje de usuarios en redes sociales y aplicaciones de comunicación son: Facebook con el 97,9\%, WhatsApp con el 33,1\%, Twitter con el 20,4\%, Skype con 13,5\%, YouTube $12.1 \%$.

En Sudamérica el uso de la Internet y redes sociales se ha incrementado, 
logrando así que del 2014 al 2016, se incrementó un $57 \%$, el uso de dichas aplicaciones, por ello la nueva cultura digital implica que Internet sea parte de la vida cotidiana, mientras que las TIC se conviertan en herramientas para desarrollar actividades como la compra y venta digital.

En Latinoamérica los usuarios conectados a la Internet hasta mediados del 2017 son 404.269.163, el crecimiento en el 2017 es de $2.137,4 \%$ y los suscriptores en Facebook son 257.242.500 usuarios activos.

Ecuador tiene una población aproximada de 16,625,776 habitantes y a inicios del 201713.471 .736 habitantes usan redes sociales, esto quiere decir que tiene el $81.0 \%$ de penetración y los suscriptores en Facebook son 9.700 .000 usuarios activos, con una tasa de penetración de $58.3 \%$.

A nivel mundial, las Redes Sociales han tomado espacios importantes en el uso diario de las personas, por lo cual se puede especificar que son un medio de información, aprendizaje, distracción y comunicación. Es por ello que más del 90 \% está conectados a una red social, el $80 \%$ de internautas visitan YouTube, un $85 \%$ de jóvenes hace las tareas escolares basados en alguna página web, un $75 \%$ usan Internet para buscar información, un $50 \%$ escucha música y un $60 \%$ de jóvenes juega en la web. 


\section{BIBLIOGRAFÎA}

Acosta, Eva. (2017). 4,5 millones de ecuatorianos son usuarios de las redes sociales. Tomado de: https:// www.metroecuador.com.ec/ec/ noticias/2017/02/15/45-millones-de-ecuatorianos-son-usuarios-de-las-redes-sociales.html

Armañanzas, E., Díaz, J., \& Meso, K. (1996). El periodismo electrónico: información y servicios multimedia en la era del ciberespacio. Barcelona.

Campos, F. (25 de mayo de 2013). Revistas Científicas Complutenses, 2468. Las redes sociales trastocan los modelos de los medios de comunicación tradicionales.

Foon Hew, K. (2011). Students' and teachers' use of Facebook

García Muñiz, A. S., \& Ramos Carvajal, C. (2003). Las redes sociales como herramienta de análisis estructural input-output. REDES. Revista hispana para el análisis de redes sociales, (4).

Guaña-Moya, E. J., Quinatoa-Arequipa, E., \& Pérez-Fabara, M. A. (2017). Ten- dencias del uso de las tecnologías y conducta del consumidor tecnológico. Ciencias Holguín, 23(2).

INEC (2017). Censo Nacional Económico, recuperado el 03 de mayo de 2017, tomado de http://www. ecuadorencifras.gob.ec/censo-nacional-economico/

Morduchowicz, R., MARCoN, A., Sylvestre, V., \& Ballestrini, F. (2012). Los adolescentes y las redes sociales. La construcción de la identidad juvenil en Internet. Buenos Aires: FCE.

Moya, E. J. G., Escobar, A. G. A., \& Remache, K. J. O. (2015). Caracterización del consumidor digital ecuatoriano. Revista Publicando, 2(5), 226242.

Piscitelli A, Adaime I, Binder I. (2010). El proyecto Facebook y la posuniversidad.

Stats, I. W. (2017). "Internet world stats: Usage and population statis TIC. Retrieved from Internet World Stats": $\quad$ http://www.Internetworldstats.com/stats.htm. [consultado 18/09/2017] 